\title{
Novel modes of non-invasive ventilation in chronic respiratory failure: a narrative review
}

\author{
Neeraj Mukesh Shah ${ }^{1,2,3}$, Rebecca F. D'Cruz ${ }^{1,2,3}$, Patrick B. Murphy ${ }^{1,2,3}$ \\ ${ }^{1}$ Lane Fox Respiratory Service, St Thomas' Hospital, Guy's and St Thomas' NHS Foundation Trust, London, UK; ${ }^{2}$ Lane Fox Clinical Respiratory \\ Physiology Centre, Guy's and St Thomas' NHS Foundation Trust, London, UK; ${ }^{3}$ Centre for Human and Applied Physiological Sciences (CHAPS), \\ King's College London, London, UK \\ Contributions: (I) Conception and design: PB Murphy; (II) Administrative support: All authors; (III) Provision of study materials or patients: All \\ authors; (IV) Collection and assembly of data: All authors; (V) Data analysis and interpretation: All authors; (VI) Manuscript writing: All authors; (VII) \\ Final approval of manuscript: All authors. \\ \#These authors contributed equally to this work. \\ Correspondence to: Patrick B. Murphy. Lane Fox Respiratory Unit, St. Thomas Hospital, Westminster Bridge Road, London, SE1 7EH, London, UK. \\ Email: patrick.murphy@gstt.nhs.uk.
}

\begin{abstract}
Home non-invasive ventilation (NIV) is central in the management of chronic hypercapnic respiratory failure and is associated with improvements in clinically relevant outcomes. Home NIV typically involves delivery of fixed positive inspiratory and expiratory airway pressures. These pressures do not reflect physiological changes to respiratory mechanics and airway calibre during sleep, which may impact on physiological efficacy, subsequent clinical outcomes, and therapy adherence. Novel ventilator modes have been designed in an attempt to address these issues. Volume-assured pressure support modes aim to automatically adjust inspiratory pressure to achieve a pre-set target tidal volume. The addition of autotitrating expiratory pressure to maintain upper airway calibre is designed for patients at risk of upper airway collapse, such as obese patients and those with obstructive sleep apnoea complicating their hypercapnic failure. Heterogeneity in setup protocols, patient selection and trial design limit firm conclusions to be drawn on the clinical efficacy of these modes. However, there are data to suggest that compared to fixedpressure NIV, volume-assured modes may improve nocturnal carbon dioxide, sleep quality and ventilator adherence in select patients. The use of the forced oscillation technique to identify expiratory flow limitation and adjust expiratory pressure to eliminate it is the most recent addition to these advanced modes and is yet to be assessed in formal clinical trials.
\end{abstract}

Keywords: Non-invasive ventilation (NIV); chronic respiratory failure; domiciliary ventilation; auto-titrating; pressure-targeted; volume-targeted

Submitted Jul 04, 2020. Accepted for publication Sep 22, 2020.

doi: $10.21037 /$ jtd-cus-2020-013

View this article at: http://dx.doi.org/10.21037/jtd-cus-2020-013

\section{Introduction}

Home non-invasive ventilation (NIV) is a widely used therapeutic intervention in patients with chronic hypercapnic respiratory failure (1). There is increasing evidence that it improves clinically important outcomes in patients with chronic respiratory failure secondary to neuromuscular disease, obesity related respiratory failure and chronic obstructive pulmonary disease (COPD) (2-5).
The technology used to deliver home NIV has dramatically evolved in recent years, with volume targeted ventilators largely replaced by pressure pre-set ventilators, due to improved patient comfort and leak compensation (6). There have also been advances in patient interfaces to improve patient comfort and adherence. Ventilators themselves have been developed to increase the amount and refine the type of data stored and how this is transferred using hard 


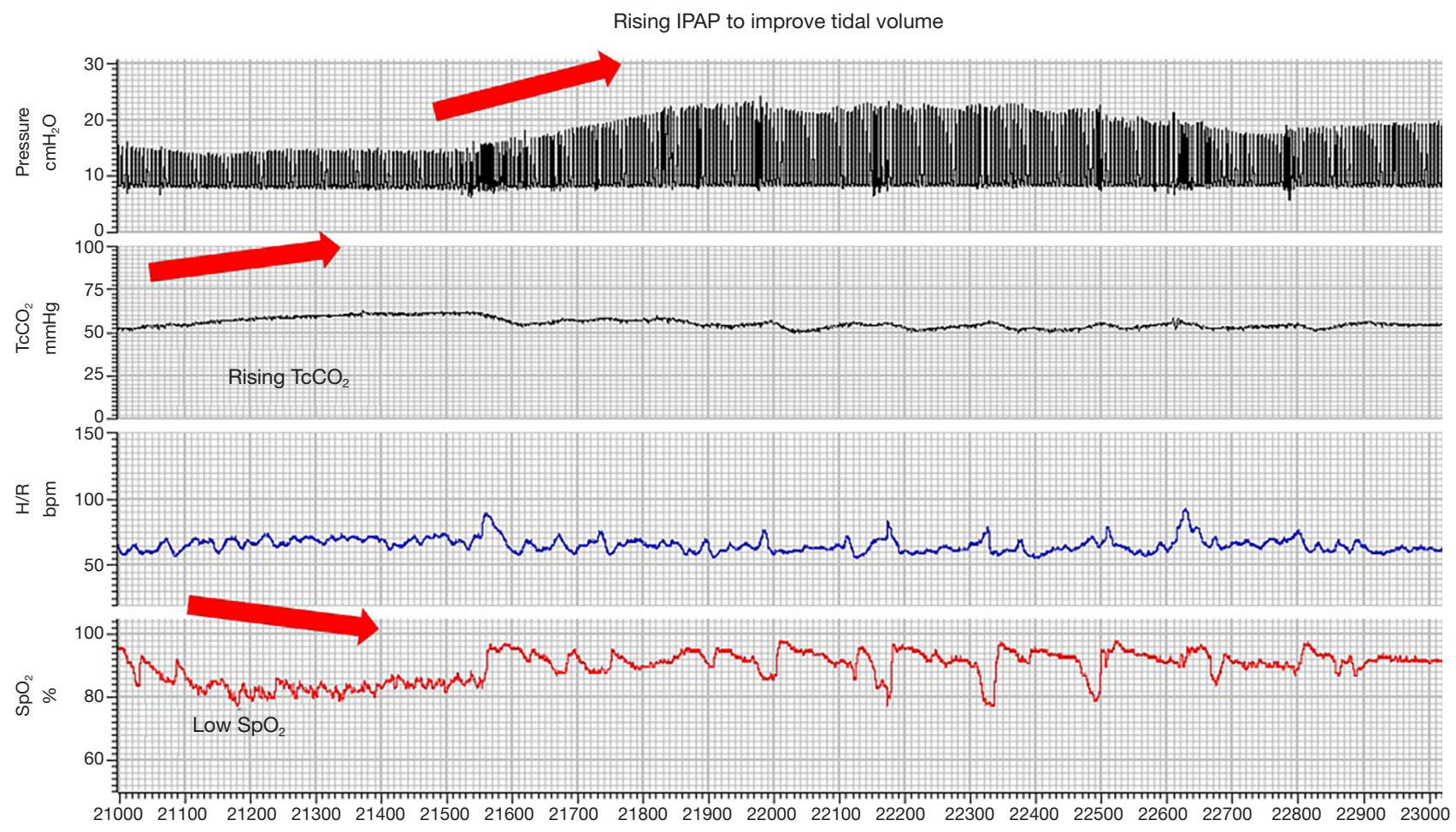

Figure 1 Extract of respiratory polygraphy demonstrating variable delivered pressure support in response to presumed hypoventilation (indicated by desaturation and rising $\mathrm{TcCO}_{2}$ ). IPAP, inspiratory positive airway pressure; H/R, heart rate; $\mathrm{SpO}_{2}$, oxy-haemoglobin saturation; $\mathrm{TcCO}_{2}$, transcutaneous carbon dioxide.

storage or wireless transfer (7). There have also been a number of novel modes of ventilation developed to improve the delivery of home NIV (Table 1). The management of obstructive sleep apnoea (OSA) has been revolutionised by the development of auto-titrating positive airway pressure (APAP) devices $(8,9)$. APAP technology has allowed the transition from a largely in-patient based setup strategy to one principally managed in an out-patient setting in many health care settings. This review will examine the available evidence for the use of auto-titrating modes of NIV in the management of patients with chronic respiratory failure.

We present the following article in accordance with the Narrative Review Checklist (available at http://dx.doi. org/10.21037/jtd-cus-2020-013).

\section{Methods}

Literature for this narrative was identified using the following terms in Medline and Embase: NIV, respiratory failure, domiciliary, auto-titrating, pressure-targeted and volume-targeted. There were no restrictions on dates or study design. English language articles were included.

\section{Technology of target volume \& ventilation}

Medical device terminology is not standardised and there is a range of terms used by different manufacturers to describe NIV modes designed to deliver variable pressure support and maintain either delivered tidal volume or alveolar ventilation. The most commonly used volume targeted modes are average volume-assured pressure support (AVAPS; Philips, Murrysville, PA, USA) and intelligent volume-assured pressure support (iVAPS; ResMed, Sydney, NSW, Australia). These modes target tidal volume and alveolar ventilation, respectively. Both systems require clinicians to input desired targets for tidal volume or ventilation alongside maximum and minimum ranges for the pressures delivered to achieve this. The devices estimate patient tidal volume and ventilation and adapt the delivered pressure within the prescribed range (Figure 1). It must therefore be realised that if pressure ranges are set incorrectly, the device may not be able to 
deliver the pressures required to achieve the pre-set targets and hypo- or hyper-ventilation may occur. Furthermore, the algorithms rely on the assumption that the estimation of patient tidal volume and ventilation is accurate. The accuracy of this and other aspects of the ventilator derived data has yet to be validated and therefore needs careful evaluation when delivering in clinical practice (10). Furthermore, it is important that when calculating target tidal volume that ideal rather than actual body weight is used.

Following the advent of hybrid pressure-volume modes of NIV which actively alter the inspiratory positive airway pressure (IPAP), technological advances have occurred in the delivery of expiratory positive airway pressure (EPAP) to either control upper airway occlusion occurring secondary to sleep apnoea (AVAPS-AE, or iVAPS with auto-EPAP) or to offset expiratory flow limitation (EFL). The use of flowbased algorithms that are used in APAP devices are not appropriate for patients with NIV due to the higher flows delivered. Newer strategies including the use of forced oscillatory technique has been developed to overcome these issues $(11,12)$.

\section{Control of hypoventilation}

Sleep related hypoventilation is a hallmark of chronic respiratory failure, with the augmentation of alveolar ventilation by NIV representing a main therapeutic target of management. Sleep represents a physiological stress for the respiratory system with changes in the load-capacitydrive relationship of the respiratory system. There is a physiological fall in tidal volume with a corresponding increase in arterial carbon dioxide occurring with the transition from wakefulness through light sleep into slow-wave and rapid-eye-movement sleep stages (13). The conventional approach of providing a fixed level of pressure support on NIV does not adapt to these changes in ventilatory need. The development of target volume modes of NIV looked to address this discrepancy and better match pressure support with patient need over the duration of a night (14). A randomized study comparing standard NIV with AVAPS in stable hypercapnic COPD patients reported a more significant improvement in daytime arterial partial pressure of oxygen $\left(\mathrm{PaCO}_{2}\right)$ following six months of AVAPS therapy. This was accompanied by a more significant increase in health-related quality of life and exercise tolerance (15). Similarly, a randomised study comparing standard NIV with iVAPS has reported a greater decrease in transcutaneous carbon dioxide $\left(\mathrm{TcCO}_{2}\right)$ with iVAPS (16). Conversely, other randomised studies comparing usual NIV with AVAPS (17) or iVAPS (18) failed to demonstrate any added benefit of the advanced modes on $\mathrm{PaCO}_{2}$. A study investigating AVAPS in stable obesity hypoventilation patients (OHS) reported a larger improvement in mean nocturnal $\mathrm{PaCO}_{2}$ than standard NIV (19), while a retrospective case-control reported improvement in daytime $\mathrm{PaCO}_{2}$ in patients with OHS and COPD-OSA overlap syndrome only (20). The data in patients with neuromuscular diseases are equally contradictory. A randomised trial comparing an auto-titrating ventilator with usual NIV in a broad spectrum of patients with neuromuscular conditions and chest wall disease reported that mean overnight $\mathrm{TcCO}_{2}$ was higher with the autotitrating device (21). On the other hand, a real world singlenight split titration study in patients with amyotrophic lateral sclerosis reported that mean nocturnal $\mathrm{TcCO}_{2}$ was lower during the AVAPS period compared to the usual NIV period (22). Interpretation of the available data is challenging as the comparison of auto-titrating modes to fixed level pressure support can be misleading if the setup strategies are not adequately controlled. For example, Storre et al. (19) demonstrated superior control of nocturnal hypoventilation using a target volume mode, however the study setup led to a higher mean delivered pressure support during ventilation with the novel mode compared to fixed level pressure support. The increased level of pressure support delivered with these modes can lead to increased sleep disruption and therefore needs careful assessment during patient setup and is discussed in more detail later in this review (23). There are several methods of achieving an automated control of hypoventilation: using variable pressure support based on estimated target volume, or by adjusting the ventilator respiratory rate. The automated adjustment of respiratory rate may be aimed at optimising patient triggered breaths, thus presumed to improve comfort, or to maximise ventilator delivered breaths to provide a more controlled mode of ventilation, and presumed improvement in central chemosensitivity (24). There are few reliable data to demonstrate superiority of either strategy, with no head to head comparisons available. Therefore, the results using any specific device and algorithm cannot be extrapolated as a class effect and the clinician must choose a mode dependent on the patients underlying condition and physiological impairment. The chosen mode requires careful evaluation to ensure adequate correction of sleep disordered breathing with titration 
Table 1 Novel modes of ventilation, advantages, and disadvantages

\begin{tabular}{|c|c|c|c|}
\hline Mode & Description & Advantage & Disadvantage \\
\hline APAP & $\begin{array}{l}\text { Commonest method uses flow } \\
\text { wave analysis to maintain upper } \\
\text { airway patency }\end{array}$ & $\begin{array}{l}\text { - Reduces need for attended polygraphy } \\
\text { - Cost effective }\end{array}$ & \\
\hline AVAPS & $\begin{array}{l}\text { - Fixed EPAP with variable IPAP to } \\
\text { achieve pre-set tidal volume }\end{array}$ & $\begin{array}{l}\text { - Compensation of change in body position } \\
\text { and disease progression }\end{array}$ & $\begin{array}{l}\text { - Accuracy of the estimation of } \\
\text { tidal volume and ventilation is } \\
\text { unclear }\end{array}$ \\
\hline \multirow[t]{2}{*}{ iVAPS } & - Bi-level PAP & - Superior control of sleep hypoventilation & $\begin{array}{l}\text { Requires a prescribed range of } \\
\text { pressures, which need to be set } \\
\text { correctly }\end{array}$ \\
\hline & $\begin{array}{l}\text { - Fixed EPAP with variable IPAP to } \\
\text { achieve pre-set tidal volume } \\
\text { - Variable backup rate designed to } \\
\text { maximise patient triggering }\end{array}$ & $\begin{array}{l}\text { - Compensation of change in body position } \\
\text { and disease progression } \\
\text { - Possible improvement in daytime } \mathrm{PaCO}_{2}\end{array}$ & $\begin{array}{l}\text { - Accuracy of the estimation of } \\
\text { tidal volume and ventilation is } \\
\text { unclear }\end{array}$ \\
\hline $\begin{array}{l}\text { AVAPS-AE or } \\
\text { iVAPS with } \\
\text { auto-EPAP }\end{array}$ & - Bi-level PAP as described above & $\begin{array}{l}\text { - Adjusts EPAP in respond to changes in } \\
\text { upper airway calibre during sleep }\end{array}$ & $\begin{array}{l}\text { - Only short-term data available } \\
\text { on efficacy }\end{array}$ \\
\hline
\end{tabular}

APAP, auto-titrating positive airway pressure; AVAPS, average volume-assured pressure support; iVAPS, intelligent volume-assured pressure support; AVAPS-AE, average volume-assured pressure support with auto-EPAP; EPAP, expiratory positive airway pressure; OSA, obstructive sleep apnoea.

against objective markers, such as $\mathrm{TcCO}_{2}$.

\section{Control of upper airway obstruction}

Obesity is an increasingly common co-morbidity in patients with chronic respiratory failure and may contribute to development of respiratory failure directly or indirectly (25). As rates of obesity increase in the population, the proportion of patients with OSA complicating their chronic respiratory failure has also increased (26). It has therefore become increasingly important to phenotype sleep disordered breathing so that appropriate correction of upper airway obstruction can be achieved with the delivery of home NIV. Analogous to the setting of pressure support during NIV setup, the level of EPAP required to control upper airway obstruction has largely been fixed following a single night titration. This approach does not account for changes that may occur with sleep stage and position in the short term or with disease progression, weight change and medication in the longer term. The AVAPS-auto EPAP (AVAPS-AE) and iVAPS-auto EPAP modes of ventilation aim to not only deliver volume-assured pressure support, but also to adjust EPAP in order to maintain upper airway patency. This mode of ventilation is seldom reported in the literature. A small randomised study comparing usual NIV with AVAPS-AE in COPD-OSA overlap syndrome failed to demonstrate any additional improvement in mean nocturnal $\mathrm{TcCO}_{2}$ with AVAPS-AE therapy after six weeks, 
but subjective sleep comfort and ventilator adherence were significantly better (27). Other studies comparing standard NIV with AVAPS-AE (28) and iVAPS-manual EPAP with iVAPS-auto EPAP $(29,30)$ reported non-inferiority of the advanced modes to standard NIV, and have demonstrated adequate safety in controlling upper airway obstruction in patients with neuromuscular disease, OHS and COPD. They did, however, fail to demonstrate any significant clinical advantages of the advanced modes. When reviewing the data from these studies it can be seen that there is a trend towards delivery of a higher EPAP by the automated modes than in the fixed level system. Interestingly this has not been associated with any decrease in patient reported comfort or decrease in adherence $(27,30)$. The higher EPAP and associated reduction in upper airway obstruction may improve ventilator triggering and the efficiency of mechanical ventilation by minimising wasted pressure support which is otherwise delivered to a closed airway. The technology used to assess upper airway patency can also be modified to assess for expiratory flow limitation (EFL). The degree of EFL varies overnight, depending upon sleep position (31) and patients suffer from increased subjective dyspnoea if insufficient EPAP levels are delivered to offset EFL (32). Therefore, a device that can measure breath-bybreath EFL and automatically adjust EPAP may result in improved dyspnoea and/or ventilator adherence. Devices which incorporate the forced oscillation technique (FOT) to detect EFL have been shown in physiological studies to minimise transdiaphragmatic pressure swings and neural respiratory drive in patients with COPD (32). The results of clinical trials evaluating FOT-based auto-titrating devices are eagerly awaited.

\section{Sleep disruption, patient comfort $\&$ adherence}

The use of variable modes of ventilation raised the concern that changes in pressure support to maintain ventilation will induce arousals that worsen sleep quality. Whilst there were early data suggesting this may be the case (23), it has subsequently been attributed to the specific setup strategy used in the study which led to supra-physiological target volumes and thus delivered pressure support. Subsequent data have demonstrated stable sleep architecture and objective sleep parameters in COPD (14) and OHS $(19,28)$ and improved subjective sleep quality $(16,17,27)$. In patients with Amyotrophic Lateral Sclerosis (ALS), arousal index was lower during the AVAPS phase of the single night study (22). There are limited data suggesting that these novel modes improve patient comfort with subsequent improvement in patient adherence $(27,33)$. However, similar to data in patients with OSA using APAP, improved compliance has not been shown to translate into improved clinical outcomes.

\section{Novel patient pathways}

The use of automated ventilator technology offers the potential to improve the traditional patient pathways for establishing NIV. A study comparing AVAPS-AE with usual NIV reported a significant decrease in NIV setup duration from four days with usual NIV to three days with AVAPS-AE (28). Given that these patients are often admitted to high-acuity beds, a reduction of length of stay by $25 \%$ may result in significant cost savings. This technology also has the potential to eliminate the need for inpatient set-up of NIV entirely. This has been examined in the outpatient vs inpatient setup of NIV in OHS (OPIP) trial $(34,35)$. The study examined the use of an auto-titrating NIV device with target volume, autoEPAP and auto-back up rate modes. The outpatient arm utilised the novel device whilst the inpatient arm used the gold standard approach of an attended respiratory sleep study and overnight titration. The study was designed to evaluate both safety (change in daytime $\mathrm{PaCO}_{2}$ ) as well as cost-effectiveness of this strategy. The study was conducted in the UK and France and showed similar clinical efficacy and cost-effectiveness of both approaches. Interestingly the patients established on home NIV via the outpatient pathway had higher subsequent contact with clinical staff than those setup via an inpatient admission. This is consistent with other home and outpatient NIV setup studies and offsets some of the cost reduction from avoiding an inpatient admission (36). Auto-NIV modes may also be used for ongoing follow-up, evaluation and optimisation of patients established on home NIV. This practice approach was examined by Mansell et al. who evaluated the use of an auto-NIV and period of remote monitoring after a clinical review in order to optimise home NIV in a pre-post intervention design (37). The evaluation of 52 patients indicated the safety of this approach with patients having a small increase in compliance following the intervention [\% days $>4$ hours $90 \%$ to $96 \%(\mathrm{P}=0.007)$ and compliance 6.5 hours/night to 6.9 hours/night $(\mathrm{P}=0.211)$ ]. However, the clinical relevance of a change of this magnitude is debatable and the costeffectiveness of such strategies requires further evaluation. 


\section{Conclusions}

Whilst there remains empirical appeal to the use of automated modes of NIV it is important to understand the underlying algorithms used to drive the technology when implementing them in clinical practice. It is also important that the modes are not substituted for clinical assessment by a trained health care professional. The delivery of home NIV requires assessment of physiological and clinical parameters to ensure the NIV prescription adequately corrects sleep disordered breathing and stabilises or reverses chronic respiratory failure.

\section{Acknowledgments}

Funding: None.

\section{Footnote}

Provenance and Peer Review: This article was commissioned by the Guest Editor (Joerg Steier) for the series " 5 th Clinical Update Sleep" published in Fournal of Thoracic Disease. The article was sent for external peer review.

Reporting Checklist: The authors have completed the Narrative Review Checklist. Available at http://dx.doi. org/10.21037/jtd-cus-2020-013

Conflicts of Interest: All authors have completed the ICMJE uniform disclosure form (available at http://dx.doi. org/10.21037/jtd-cus-2020-013). The series " $5^{\text {th }}$ Clinical Update Sleep" was commissioned by the editorial office without any funding or sponsorship. PBM reports grants and personal fees from Philips, grants, and personal fees from ResMed, grants and personal fees from F\&P, grants, and personal fees from B\&D Electromedical, personal fees from Santhera, grants from GSK, outside the submitted work. The authors have no other conflicts of interest to declare.

Ethical Statement: The authors are accountable for all aspects of the work in ensuring that questions related to the accuracy or integrity of any part of the work are appropriately investigated and resolved.

Open Access Statement: This is an Open Access article distributed in accordance with the Creative Commons Attribution-NonCommercial-NoDerivs 4.0 International
License (CC BY-NC-ND 4.0), which permits the noncommercial replication and distribution of the article with the strict proviso that no changes or edits are made and the original work is properly cited (including links to both the formal publication through the relevant DOI and the license). See: https://creativecommons.org/licenses/by-nc-nd/4.0/.

\section{References}

1. Patout M, Ramsay M, Mackie M, et al. Home mechanical ventilation (HMV): Setup and outcome in Europe. Eur Resp J 2015;46:OA4780.

2. Murphy PB, Rehal S, Arbane G, et al. Effect of Home Noninvasive Ventilation With Oxygen Therapy vs Oxygen Therapy Alone on Hospital Readmission or Death After an Acute COPD Exacerbation: A Randomized Clinical Trial. JAMA 2017;317:2177-86.

3. Bourke SC, Tomlinson M, Williams TL, et al. Effects of non-invasive ventilation on survival and quality of life in patients with amyotrophic lateral sclerosis: a randomised controlled trial. Lancet Neurol 2006;5:140-7.

4. Marin JM, Soriano JB, Carrizo SJ, et al. Outcomes in patients with chronic obstructive pulmonary disease and obstructive sleep apnea: the overlap syndrome. Am J Respir Crit Care Med 2010;182:325-31.

5. Masa JF, Corral J, Alonso ML, et al. Efficacy of Different Treatment Alternatives for Obesity Hypoventilation Syndrome. Pickwick Study. Am J Respir Crit Care Med 2015;192:86-95.

6. Windisch W, Storre JH, Sorichter S, et al. Comparison of volume- and pressure-limited NPPV at night: a prospective randomized cross-over trial. Respir Med 2005;99:52-9.

7. Borel JC, Palot A, Patout M. Technological advances in home non-invasive ventilation monitoring: Reliability of data and effect on patient outcomes. Respirology 2019;24:1143-51.

8. Berry RB, Hill G, Thompson L, et al. Portable monitoring and autotitration versus polysomnography for the diagnosis and treatment of sleep apnea. Sleep 2008;31:1423-31.

9. Patil SP, Ayappa IA, Caples SM, et al. Treatment of Adult Obstructive Sleep Apnea With Positive Airway Pressure: An American Academy of Sleep Medicine Systematic Review, Meta-Analysis, and GRADE Assessment. J Clin Sleep Med 2019;15:301-34.

10. Ambrosino N, Vitacca M, Dreher M, et al. Telemonitoring of ventilator-dependent patients: a European Respiratory Society Statement. Eur Respir J 
2016;48:648-63.

11. Dellaca RL, Rotger M, Aliverti A, et al. Noninvasive detection of expiratory flow limitation in COPD patients during nasal CPAP. Eur Respir J 2006;27:983-91.

12. Zannin E, Chakrabarti B, Govoni L, et al. Detection of Expiratory Flow Limitation by Forced Oscillations during Noninvasive Ventilation. Am J Respir Crit Care Med 2019;200:1063-5.

13. Douglas NJ, White DP, Pickett CK, et al. Respiration during sleep in normal man. Thorax 1982;37:840-4.

14. Ambrogio C, Lowman X, Kuo M, et al. Sleep and noninvasive ventilation in patients with chronic respiratory insufficiency. Intensive Care Med 2009;35:306-13.

15. Magdy DM, Metwally A. Effect of average volume-assured pressure support treatment on health-related quality of life in COPD patients with chronic hypercapnic respiratory failure: a randomized trial. Respir Res 2020;21:64.

16. Ekkernkamp E, Storre JH, Windisch W, et al. Impact of Intelligent Volume-Assured Pressure Support on Sleep Quality in Stable Hypercapnic Chronic Obstructive Pulmonary Disease Patients: A Randomized, Crossover Study. Respiration 2014;88:270-6.

17. Crisafulli E, Manni G, Kidonias M, et al. Subjective Sleep Quality During Average Volume Assured Pressure Support (AVAPS) Ventilation in Patients with Hypercapnic COPD: A Physiological Pilot Study. Lung 2009;187:299.

18. Oscroft NS, Chadwick R, Davies MG, et al. Volume assured versus pressure preset non-invasive ventilation for compensated ventilatory failure in COPD. Respir Med 2014;108:1508-15.

19. Storre JH, Seuthe B, Fiechter R, et al. Average volumeassured pressure support in obesity hypoventilation: A randomized crossover trial. Chest 2006;130:815-21.

20. Horvath CM, Brutsche MH, Baty F, et al. iVAPS (intelligent volume assured pressure support) for noninvasive ventilation - a case-control study. Eur Respir J 2014;44:P4702.

21. Jaye J, Chatwin M, Dayer M, et al. Autotitrating versus standard noninvasive ventilation: a randomised crossover trial. Eur Respir J 2009;33:566-71.

22. Patel Salma I, Gay P, Morgenthaler Timothy I, et al. Practical Implementation of a Single-Night SplitTitration Protocol With BPAP-ST and AVAPS in Patients With Neuromuscular Disease. J Clin Sleep Med 2018;14:2031-5.

23. Janssens JP, Metzger M, Sforza E. Impact of volume targeting on efficacy of bi-level non-invasive ventilation and sleep in obesity-hypoventilation. Respir Med
2009;103:165-72.

24. Murphy PB, Davidson C, Hind MD, et al. Volume targeted versus pressure support non-invasive ventilation in patients with super obesity and chronic respiratory failure: a randomised controlled trial. Thorax 2012;67:727-34.

25. Shah NM. Hypercapnic Failure in Non-COPD. In: Heunks L, Demoule A, Windisch W. Pulmonary Emergencies. ERS Monograph: ERS, 2016:86-100.

26. Balachandran JS, Masa JF, Mokhlesi B. Obesity Hypoventilation Syndrome Epidemiology and Diagnosis. Sleep Med Clin 2014;9:341-7.

27. Murphy PB, Arbane G, Ramsay M, et al. Safety and efficacy of auto-titrating noninvasive ventilation in COPD and obstructive sleep apnoea overlap syndrome. Eur Respir J 2015;46:548-51.

28. Patout M, Gagnadoux F, Rabec C, et al. AVAPS-AE versus ST mode: A randomized controlled trial in patients with obesity hypoventilation syndrome. Respirology 2020. [Epub ahead of print].

29. Orr JE, Coleman J, Criner GJ, et al. Automatic EPAP intelligent volume-assured pressure support is effective in patients with chronic respiratory failure: A randomized trial. Respirology 2019;24:1204-11.

30. McArdle N, Rea C, King S, et al. Treating Chronic Hypoventilation With Automatic Adjustable Versus Fixed EPAP Intelligent Volume-Assured Positive Airway Pressure Support (iVAPS): A Randomized Controlled Trial. Sleep 2017. doi: 10.1093/sleep/zsx136.

31. Milesi I, Porta R, Vitacca M, et al. Overnight monitoring of lung mechanics and Tidal expiratory flow limitation (EFLT) by Forced Oscillation Technique (FOT) in Chronic Obstructive Pulmonary Disease (COPD) receiving non-invasive ventilation (NIV): the impact of sleep and posture. Eur Respir J 2018;52:PA2380.

32. Suh ES, Pompilio P, Mandal S, et al. Auto-titrating external positive end-expiratory airway pressure to abolish expiratory flow limitation during tidal breathing in patients with severe chronic obstructive pulmonary disesase: a physiological study. Eur Respir J 2020;56:1902234.

33. Storre JH, Matrosovich E, Ekkernkamp E, et al. Home mechanical ventilation for COPD: high-intensity versus target volume noninvasive ventilation. Respir Care 2014;59:1389-97.

34. Mandal S, Arbane G, Murphy P, et al. Medium-term costeffectiveness of an automated non-invasive ventilation outpatient set-up versus a standard fixed level non-invasive ventilation inpatient set-up in obese patients with chronic 
respiratory failure: a protocol description. BMJ Open 2015;5:e07082.

35. Murphy PB, Patout M, Flach C, et al. Late Breaking Abstract - Cost-effectiveness of outpatient (OP) vs. inpatient (IP) setup of home non-invasive ventilation (NIV) in obesity hypoventilation syndrome (OHS): A Randomised Clinical Trial. Eur Respir J 2019;54:RCT5099.

36. Duiverman ML, Vonk JM, Bladder G, et al. Home

Cite this article as: Shah NM, D'Cruz RF, Murphy PB. Novel modes of non-invasive ventilation in chronic respiratory failure: a narrative review. J Thorac Dis 2020;12(Suppl 2):S217-S224. doi: $10.21037 /$ jtd-cus-2020-013 initiation of chronic non-invasive ventilation in COPD patients with chronic hypercapnic respiratory failure: a randomised controlled trial. Thorax 2020;75:244-52.

37. Mansell SK, Cutts S, Hackney I, et al. Using domiciliary non-invasive ventilator data downloads to inform clinical decision-making to optimise ventilation delivery and patient compliance. BMJ Open Respir Res 2018;5:e00238. 